Relations industrielles

Industrial Relations

\title{
Upgrading Blue Collar and Service Workers, by Charles Breckers, Policy Study in Employment and Welfare, Number 12, The John Hopkins University Press, Baltimore, 1972, 113 pp.
}

Jean Sexton

Volume 29, numéro 2, 1974

URI : https://id.erudit.org/iderudit/028523ar

DOI : https://doi.org/10.7202/028523ar

Aller au sommaire du numéro

Éditeur(s)

Département des relations industrielles de l'Université Laval

ISSN

0034-379X (imprimé)

1703-8138 (numérique)

Découvrir la revue

Citer ce compte rendu

Sexton, J. (1974). Compte rendu de [Upgrading Blue Collar and Service Workers, by Charles Breckers, Policy Study in Employment and Welfare, Number 12, The John Hopkins University Press, Baltimore, 1972, 113 pp.] Relations industrielles / Industrial Relations, 29(2), 418-419. https://doi.org/10.7202/028523ar

Tous droits réservés (C Département des relations industrielles de l'Université Laval, 1974
Ce document est protégé par la loi sur le droit d'auteur. L'utilisation des services d'Érudit (y compris la reproduction) est assujettie à sa politique d'utilisation que vous pouvez consulter en ligne.

https://apropos.erudit.org/fr/usagers/politique-dutilisation/ 
développement organisationnel en cours. C'est aussi un pas en avant dans l'élaboration d'une théorie sur l'introduction des changements au sein des organisations de travail.

\section{Université Laval}

\section{Laurent BELANGER}

Upgrading Blue Collar and Service Workers, by Charles Breckers, Policy Study in Employment and Welfare, Number 12, The John Hopkins University Press, Baltimore, 1972, 113 pp.

Le perfectionnement (upgrading) des travailleurs n'est sûrement pas un sujet nouveau. Cependant cela ne veut pas dire pour autant qu'il s'agit d'un sujet facile. En effet, différents comportements, réactions et patterns peuvent être observés dépendant du niveau d'activité économique, des pratiques d'embauche, des facilités de formation, de la participation des entreprises, etc.

Le perfectionnement de la main-d'oeuvre est partie intégrale de la planification de main-d'oeuvre et a constitué et continue à constituer une panacée pour plusieurs. Ce genre d'investissements dans le capital humain rencontre cependant plusieurs obstacles et, dans un tel contexte, il est à se demander si a priori un tel effort n'est pas voué à un échec presque complet compte tenu des objectifs (souvent imprécis) initiaux.

Ce court ouvrage traite donc de perfectionnement mais d'une façon relativement différente de celle qu'on est habitué de voir. En effet, il s'agit ici d'études de cas de perfectionnement dans cinq industries typiques de cols bleus et de travailleurs de service de la ville de New York: le vêtement, l'alimentation, les services de santé, la construction et le transport en commun. Déjà on peut identifier deux des aspects originaux de cette étude: d'abord l'auteur fait un effort particulier pour voir ce qui se passe dans différents secteurs industriels, ensuite il se concentre en partie sur les travailleurs de service débordant ainsi la tradition d'étudier surtout le travailleur du secteur manufacturier. Cet effort est d'autant plus louable que l'auteur sort des sentiers battus.

Avant même d'expliquer la façon dont cette étude a été faite, l'auteur s'attarde d'une excellente façon d'ailleurs, sur la définition même du concept d'« upgrading » et le fait si bien que le lecteur perçoit clairement la complexité réelle d'une telle expression. Partant de l'entendement populaire de ce terme (i.e. mouvement d'un individu d'un premier emploi à un second considéré meilleur pour une ou plusieurs raisons) Brecker s'efforce de l'éclaircir en utilisant, entre autres, la théorie des marchés internes de travail de Piore et Doeringer. Il aboutit finalement à définir «upgrading » comme étant le processus de mobilité occupationnelle intra-industrielle.

Le but de cette recherche est d'examiner le potentiel de dépenses publiques accrues, de faciliter le perfectionnement de travailleurs non-qualifiés. Autrement dit, est-il possible d'améliorer les pratiques de perfectionnement par une intervention de l'Etat? Pour répondre à cette question, l'auteur s'est concentré sur les cinq industries new-yorkaises ci-haut mentionnées. Ensemble, ces cinq industries représentent environ $20 \%$ de l'emploi total et $50 \%$ de l'emploi non col-blanc de New York. Sa méthodologie fait appel à de nombreux instruments et sources de données: revue de littérature, entrevue avec informateur-clef et avec représentants syndicaux et patronaux, et entrevues avec des administrateurs de programme de maind'oeuvre. En plus, l'auteur a analysé ces entrevues en parallèle avec des données provenant des dossiers nationaux sur les histoires de travail disponible à l'administration de la sécurité sociale, plus précisément pour le sous-échantillon de la région métropolitaine de New York et ce pour la période 1962-1966.

Par la suite, l'auteur présente successivement ses données et autres informations utiles pour chacune des cinq industries retenues pour étude. A l'intérieur de ces présentations, on retrouve des propos sur la vue globale de l'industrie, sur la mobilité occupationnelle, sur la formation et la mobilité occupationnelle, et sur les opportunités d'accroître le perfectionnement dans chacune des industries. Finalement, en guise de conclusion, l'auteur réfléchit sur ces politiques publiques et plus spécifiquement ces programmes de main-cloeuvre touchant le perfectionnement et l'avancement des travailleurs. Sa principale conclusion est d'accroître le réservoir de gens éligibles considérés pour le perfec- 
tionnement, d'améliorer les mécanismes de sélection, et de chercher à améliorer les salaires et les conditions de travail des employés au bas de l'échelle occupationnelle.

En résumé, il s'agit d'une étude originale très intéressante qui fournit amplement de matière à réflexion au lecteur. Comme critique, on pourrait reprocher à Brecker d'avoir présenté sa méthodologie trop vite et plus spécifiquement les limites inhérentes aux données utilisées et de s'être servi des don- nées un peu vieilles (malgré la difficulté permanente d'obtenir des données récentes) et ainsi omettre l'influence des principaux programmes de main-d'oeuvre américaine adoptée au milieu des années 60 .

Cependant, cette recherche demeure sûrement très intéressante et extrêmement enrichissante. Reste maintenant à voir si les résultats seraient encore les mêmes avec des données plus récentes.

Université Laval

Jean SEXTON

\section{PUBLICATIONS RÉCENTES RECENT PUBLICATIONS}

\section{Généralités}

«Tasks of the Social Science Departments of Higher Educational Institutions at the Present Stage » by V. Eliutin, The Soviet Review, (New York), vol. XIV, no 4, Winter 1973-74, pp. 67-93.

«Lutte de sexes et lutte de classes » par Jacques Dofny, Sociologie et sociétés, (Montréal), vol. VI, no 1, mai 1974, pp. 3-17.

«Consumer Behavior: Can Economics and Behavioral Science Converge? 》 by Francesco Nicosia, California Management Review, (Berkeley, Ca.), vol. XVI, no 2, Winter 1973, pp. 71-79.

«L'actionnariat du personnel dans les entreprises publiques » par Raymond Muzellec, Droit social, (Paris), no 3, mars 1974, pp. 85-108.

«Internal and External Functions of the Council of Economic Advisers » by Herbert Stein, Monthly Labor Review, (Washington), vol. 97, no 3, March 1974, pp. 42-43.

«Problems in Relating Theory to Practice » by Jenny Mellor, Inquiry, (Trondheim, Norway), vol. 17, no 1, Spring 1974, pp. 79-105.

"Measuring the Quality of Life» by Angus Campbell, Michigan Business Review, (Ann Arbor, Michigan), vol. XXVI, no 1, Jan. 1974, pp. 8-11.
«Is Work Still a Dirty World ? 》 by Ralph C. Wenrich, Michigan Business Review, (Ann Arbor, Michigan), vol. XXVI, no 2, March 1974, pp. 20-27.

«A General Systems Framework for Social Systems » by C.A. Laszlo, M.D. Levine and J.H. Milsum, Behavioral Science, (Louiseville, Kentucky), vol. 19, no 2, March 1974, pp. 79-93.

"Workers' Participation in Management: The German Experience » by A. Szakats, The Journal of Industrial Relations, (Berkeley, Ca.), vol. 16, no 1, March 1974, pp. 29-45.

\section{Travail et main-d'oeuvre}

«Regional Differences in Employment and Unemployment, 1957-72 » by Christopher G. Gellner, Monthly Labor Review, (Washington), vol. 97, no 3 , March 1974, pp. 15-25.

«Some Manpower Implications of Internal Labour Markets: A Note 》 by P.B. Beaumont, The Journal of Industrial Relations, Berkeley, Ca.), vol. 16, no 1, March 1974, pp. 85-88.

"Recommendations of the Symposium on 'Women's Employment and the Family 》 by A.-G. Kharchev and S.I. Golod, The Soviet Review, (New York), vol. XIV, no 4, Winter 1973-74, pp. 55-67. 